\title{
THEMATIC PAPERS \\ Strategic developments in the delivery of psychiatric services worldwide
}

\author{
David Skuse
}

Behavioural and Brain Sciences Unit, Institute of Child Health, London, UK, email d.skuse@ ucl.ac.uk
We are delighted to have a trio of papers from senior staff members of the World Health Organization (WHO) on the strategic development plans for psychiatric services in the African Region, in Latin America and the Caribbean, and in the Western Pacific. We are truly privileged to publish these valuable documents, which reflect the current state of the WHO's attempts to improve psychiatric services worldwide.

Dr Carina Ferreira-Borges reports from Africa, and draws attention to the WHO's Comprehensive Mental Health Action Plan for 2013-20. This emphasises the importance of delivering care in the community and of considering the notion of recovery. Despite the emphasis in the plan on moving away from a purely medical model to a socially responsive model of care for people with mental health problems, there has been little movement in this direction in Africa. Unfortunately, in most African states, mental health has a low priority. It is considered to be a 'small problem' compared with the burden of communicable diseases, an attitude fostered by the big donors from the West.
Dr Jorge Rodriguez reflects on the possibilities of delivering the objectives of the WHO's Comprehensive Mental Health Action Plan in Latin America and the Caribbean. He points out that the small proportion of the health budget of countries in the region that is spent on mental health is targeted largely at mental hospitals. Only two-thirds of countries in the region have a national mental health plan. There are considerable constraints and difficulties to overcome before 2020 .

Finally, Dr Xiangdong Wang reports from the Western Pacific. He draws our attention to the very high rate of suicide in this region: an astonishing one in three of all suicides globally takes place here. Encouraging news is adduced. There is recognition among health ministers that the disasters associated with the region in recent years have increased the burden of mental health problems, and that effective action is urgently required. It is gratifying to read about the initiatives that are taking root in the region, including a strategy to manage the high suicide rate and the pending transformation of mental healthcare delivery.

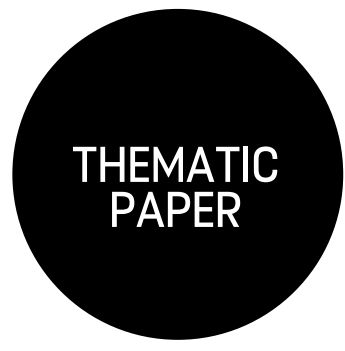

STRATEGIC DEVELOPMENTS IN THE DELIVERY OF PSYCHIATRIC SERVICES WORLDWIDE Mental health: strengthening health
and development opportunities in the
WHO African Region

\author{
Carina Ferreira-Borges
}

Technical Officer Substance Abuse, Regional Office for Africa, Congo, World Health Organization; Instituto de Higiene e Medicina Tropical, Universidade Nova de Lisboa Portugal, email ferreiraborgesc@ who.int; Ferreiraborgesc@afro. who.int

The author is a staff member of the World Health Organization The author alone is responsible The author alone is responsibews expressed in for the views expressed in
this publication and they do not necessarily represent the decisions, policy or views of the World Health Organization.
Despite the efforts of the World Health Organization (WHO) to put mental health at the core of the health and development agendas, the underdevelopment of services in Africa is widespread. The adoption of the WHO's Comprehensive Mental Health Action Plan 2013-20 at the 66th World Health Assembly in May 2013 provides an opportunity to reshape policy. In the African Region, mental health generally appears to be of low interest and people with mental health conditions are not prioritised. This article describes factors that contribute to this low level of interest and recommends collective action to prioritise mental health on the public health and social development agendas. It is also a call to all stakeholders to increase financial investment and bring mental health out of the shadows.

Research has shown that addressing mental health has a positive impact not only on the overall burden of diseases but also on human development, including economic growth and poverty and conflict reduction. The World Health Organization (WHO) has played an active role in 
putting mental health at the core of health and development agendas. This has been reflected in important global initiatives, such as the World Health Report dedicated to mental health (WHO, 2001), the 2010 WHO report Mental Health and Development, and the 2013-20 Mental Health Action Plan recently approved by the World Health Assembly (WHO, 2013). Several other initiatives, including the WHO MIND project and the Mental Health Gap Action Programme (mhGAP) have also been launched by the WHO in an attempt to increase political commitment and the capacity of member states to address mental health (WHO, 2008). Ongoing projects and initiatives have provided a better assessment of the key components of national mental health systems and so help to improve planning (WHO, 2009).

Yet, despite all the evidence showing some improvements, reforming mental health systems has not been easy in the African Region. Mental illness is not receiving from government authorities and donors the visibility, commitment and resources that are warranted by the magnitude of the burden. In the African Region, neuropsychiatric conditions make a substantial independent contribution to the burden of disease, accounting, in 2004 , for $5 \%$ of the total burden of disease in Africa (WHO, 2004), and the overall economic costs are very high. The financial and human resources available for mental healthcare in Africa are insufficient to address the problem effectively. Seventy per cent of African countries spend less than $1 \%$ of their small health budgets on mental health (WHO, 2005) and most of that is consumed by large psychiatric institutions, contrary to growing evidence for cost-effective community-based interventions (Patel et al, 2007). In the region, there is 1 psychiatrist per 2.5 million people, 1 psychiatric nurse per 500000 people and 1 psychologist per 2 million people, while the world median numbers are 1.3 psychiatrists per 100000 population, 5.8 nurses per 100000 population and 1 psychologist per 300000 population (WHO, 2011) .

An evaluation of the Regional Strategy for Mental Health 2000-10, covering 34 countries (WHO Regional Office for Africa, 2000; Lund et $a l, 2010)$, revealed a marginal increase in the proportion of African countries with a national mental health policy and mental health laws, as well as a critical shortage of financial and human resources. Although efforts are being made, the underdevelopment of mental health services in Africa is widespread. Many reasons can be advanced to explain why countries are responding in such a way to mental health problems. A study developed under the Mental Health and Poverty Project (MHaPP) in four countries of the African Region (Flisher et al, 2007; Bird et al, 2011) looked at factors affecting the low priority given to mental health and grouped them into three categories adopted from the Hall model (Hall et al, 1975). This model looks at the setting of health priorities and argues that a policy issue comes onto the policy agenda only when the issue and its possible solution are ranked high in terms of their legitimacy, feasibility and support. In a simple way, this would mean that for mental health to be prioritised in countries' policy agendas there would need to be a 'perceived social obligation' to do something to resolve the problem, a 'perceived feasibility of implementation of a response' (translated into the availability of theoretical and technical knowledge, human and financial resources and infrastructure) and 'perceived support' (translated into public support for government action, for mental health and for the proposed solutions).

Based on this study, in order to analyse the situation in the region, we have examined each of these criteria against regional findings.

\section{Legitimacy or perceived social obligation to act}

The results from the MHaPP study showed that there was a limited appreciation of the prevalence of mental illness among decision makers in the four participating countries (Ghana, South Africa, Uganda and Zambia). Several factors contributing to this situation were identified in the evaluation report, and were summarised as country-specific focal points. There was a low level of research activity at the country level due to lack of capacity, resources and funding, and lack of routine data collection. A low level of reporting from health management information systems definitely contributes to the 'invisibility' of the problem in the African Region. Mental health is considered a 'small' problem, especially when compared with many communicable diseases. The latter are extremely well documented and receive high visibility from high-profile donors. In contrast, mental health is not allocated enough financial or human resources.

Data were collected in seven countries in the African Region using the WHO Assessment Instrument for Mental Health Systems. This found that in these countries, between February 2005 and February 2008, the level of government mental health spending per capita was a median of US $\$ 0.01$ while the median level of mental health spending per capita in the other reporting countries in regions outside Africa was US\$0.30 (WHO, 2009). Such a low level of investment affects key components of each country's mental health system. It implies a very low level of service delivery and availability of drugs and human resources, and very limited capacity and information systems.

\section{Perceived feasibility of implementation of a response}

Results from the MHaPP study showed that the chronicity of mental illness, the lack of appropriate response to treatment and the lack of any tangible evaluation of treatments signal to decision makers that provision for mental health management is a waste of resources. This evaluation of the regional strategy showed that it is generally believed that mental illness is more of a spiritual illness, or is attributable to supernatural causes. Either way, the 
implication is that it is not appropriately addressed by the health system. Such misconceptions, together with the poor responses to treatment or lack of data on treatment outcomes, strengthen the view of governments that mental disorders are either untreatable or that they are expensive to treat.

The current situation reveals limitations regarding current knowledge about mental health and the methods of treatment that are potentially available. It reflects cultural and social values that are not supportive of political actions favouring appropriate responses to the problem of mental disorders (prevention, treatment, rehabilitation). Mental health services remain centralised and institution-based in most countries of the region, thus consuming a lot of resources and reinforcing myths. Because of prejudicial views about mental illness, evidence of treatment impact on the outcomes of mental disorders is difficult to access. Organisational change, through decentralisation, has been advanced as an important way of improving the performance of mental health systems. There is no clear guidance, or examples of how mental healthcare can be integrated into general healthcare. There is evidence that services can be made accessible, affordable, acceptable and available, with patients being treated in their own communities rather than hospitalised far from home (WHO \& WONCA, 2008). However, the response to mental health problems in the countries surveyed tends to reinforce negative views of mental health services.

\section{Perceived support for action}

The general public's interest in the well-being of people with mental disorders is low, in marked contrast to attitudes to communicable diseases. This lack of concern is associated with a lack of funding. There is no support for mental health from big donors to global health (and to initiatives in the African Region), such as the US government, the Bill and Melinda Gates Foundation and the Global Fund, or from development partners and other agencies. That lack of interest is a barrier to progress. In a region where donor funding plays an important role in health systems and where vertical programmes are aimed at specific diseases, especially communicable diseases, developing appropriate services and providing appropriate resources are real challenges. It is striking that there are few user groups, non-governmental organisations (NGOs) or other professional associations that strongly advocate for mental health, and that deficiency contributes to low support in general.

The lack of political commitment and the low prioritisation of mental health in the allocation of resources at all levels perpetuate the slow development and implementation of mental health policies, programmes and legislation. Data from the 2010 evaluation (Lund et al, 2010) revealed that the proportion of countries with mental health policies has marginally increased, from $48 \%$ to $50 \%$, over the past few years. Although some countries do have draft policies in place, these remain inactive, as they still need to be ratified by government. Legislation faces the same constraint; only $25 \%$ of countries reporting have reformed their mental health legislation.

\section{Discussion}

The findings are largely consistent with other published studies on barriers to improved mental health (Saraceno et al, 2007; Lund et al, 2010; Bird et al, 2011). Despite efforts and some improvements, mental health continues to be left out of planning and prioritisation exercises both by governments and by other health and development stakeholders in the African Region. Competing health priorities, stigma, barriers to the implementation of policy, financial and human resource constraints and poor service delivery are both the cause and the result of this situation. Behind them lies a limited appreciation of the importance of mental health as a contributor to the overall burden of disease and poverty. This lack of appreciation has led to low levels of support for the provision of services and high levels of discrimination. People with mental disorders are seen as having a hopeless disease for which treatment represents a waste of resources, because it is largely ineffective. Alternatively, they are considered to be possessed by spirits and therefore are not ill.

On the other hand, there are some positive initiatives, developed by NGOs or by emerging users' networks for people with mental disorders, showing that change can occur and care for mental disorders can be improved (Katontoka, 2007, Basic Needs, 2012; CBM, 2013). But these are contributions that affect only a small part of the population, and they do not impact on health policy decision-making. They do not bring about the changes that are necessary for a truly public health approach to improving mental health. The recently approved 2013-20 Mental Health Action Plan is a renewed opportunity for collective action to push mental health forward on government agendas. It should help those who wish to place it on public health and social development priority agendas through coordinated and strong advocacy. It is also a call to all stakeholders to increase the priority of mental health and to call for greater financial investment, and therefore to bring mental health out of the shadows.

\section{Conclusion}

It needs to be recognised that, for many different reasons, addressing mental health in the WHO African Region represents an immense challenge. Governments play a pivotal role in bringing mental health onto the public health and social development priority agendas, but will be able to do so only if other stakeholders such as multilateral and bilateral agencies, including development agencies, donors, mental health and public health practitioners, researchers and civil society and consumers, actively support mental health intervention. 


\section{References}

Basic Needs (2012) About us. At http://www.basicneeds.org/html/ about.htm (accessed October 2013).

Bird, P., Omar, M., Doku, V., et al (2011) Increasing the priority of mental health in Africa: findings from qualitative research. Health Policy Plan, 26, 357-365.

CBM (2013) Africa. At http://www.cbm.org/Africa-252070.php (accessed October 2013).

Flisher, A., Lund, C., Funk, M., et al (2007) Mental health policy development and implementation in four African countries. Journal of Health Psychology, 12, 505-516.

Hall, P., Land, H., Parker, R., et al (1975) Change, Choice and Conflict in Social Policy. Heinemann.

Katontoka, S. (2007) Users' networks for Africans with mental disorders Lancet, 370, 919-920.

Lund, C., Heyningen, T. \& Majavu, M. (2010) Evaluation of the WHO African Regional Strategy for Mental Health 2000-2010. Report for the World Health Organization Regional Office for Africa. WHO.

Patel, V., Araya, R., Chatterjee, S., et al (2007) Treatment and prevention of mental disorders in low-income and middle-income countries. Lancet, 370, 991-1005.
Saraceno, B., van Ommeren, M., Batniji, R., et al (2007) Barriers to improvement of mental health services in low-income and middleincome countries. Lancet, 370, 1164-1174.

WHO (2001) Mental Health: New Understanding, New Hope. World Health Report. WHO

WHO (2004) Disease and Injury Estimates for 2004. WHO Available at http://www.who.int/healthinfo/global burden disease/ estimates_regional/en/index.html (accessed 15 October 2013).

WHO (2005) Mental Health Atlas 2005. WHO.

WHO (2008) Mental Health Gap Action Programme. Scaling Up Care for Mental, Neurological, and Substance Use Disorders. WHO.

WHO (2009) Mental Health Systems in Selected Low- and MiddleIncome Countries: A Who-Aims Cross-National Analysis. WHO

WHO (2011) Mental Health Atlas 2011. WHO.

WHO (2013) Comprehensive Mental Health Action Plan 2013-2020. WHO. Available at http://www.who.int/mental_health/ action_plan_2013/en (accessed December 2013)

WHO \& WONCA (2008) Integrating Mental Health into Primary Care A Global Perspective. World Health Organization and World Organization of Family Doctors.

WHO Regional Office for Africa (2000) Regional Strategy for Mental Health 2000-2010 (AFR/RC49/9). WHO.

\section{THEMATIC}

PAPER

Senior Advisor on Mental Health. Pan American Health Organization/Regional Office of the World Health Organization, Washington, DC, USA, email rodrigjo@paho.org
Mental illnesses are a growing health problem and reducing the treatment gap in Latin America and the Caribbean is a great challenge. Evaluations conducted by the Pan American Health Organization (PAHO) and World Health Organization (WHO) have shown that the responsiveness of health services is still limited. Nonetheless, from an evaluation of how mental health reform has progressed in the region following the historical benchmark of the Caracas Declaration (1990), it is clear that - despite the limitations, shortcomings and challenges - significant progress has been made in most countries. This paper briefly reviews this progress.

Mental illnesses are a growing health problem in the Americas, as in the rest of the world. In 1990, mental and neurological disorders accounted for $8.8 \%$ of the total burden of disease in Latin America and the Caribbean (LA\&C), estimated in terms of disability-adjusted life-years (DALYs).
This proportion had more than doubled, to $21 \%$, by 2006 (PAHO, 2009; Rodriguez et al, 2009a,b).

A review of the most relevant epidemiological studies of mental disorders conducted in LA\&C showed that, in recent years, the estimated average prevalence rates in the adult population (measured during the preceding year) has been $1.0 \%$ for non-affective psychoses, $4.9 \%$ for major depression and $5.7 \%$ for alcohol misuse or dependence. It also revealed that more than a third of people with non-affective psychosis, over half of those with depression and about three-quarters of those who were dependent on or misused alcohol had not received any medical treatment, from either specialised or general services (PAHO, 2009; Rodriguez et al, 2009a,b).

In practical terms, this means that only a minority of people who need mental healthcare actually receive it. To this situation must be added the fact that mental illnesses produce a high degree of disability, and they particularly affect those in the population who are most vulnerable, for whom services are scarce (PAHO, 2009; Rodriguez et al, 